\title{
Aging and a Peripheral Immune Challenge Interact to Reduce Mature Brain-Derived Neurotrophic Factor and Activation of TrkB, PLC $\gamma 1$, and ERK in Hippocampal Synaptoneurosomes
}

\author{
Giuseppe P. Cortese, Ruth M. Barrientos, Steven F. Maier, and Susan L. Patterson \\ Department of Psychology and Neuroscience, and The Center for Neuroscience, University of Colorado, Boulder, Colorado 80309
}

For reasons that are not well understood, aging significantly increases brain vulnerability to challenging life events. High-functioning older individuals often experience significant cognitive decline after an inflammatory event such as surgery, infection, or injury. We have modeled this phenomenon in rodents and have previously reported that a peripheral immune challenge (intraperitoneal injection of live Escherichia coli) selectively disrupts consolidation of hippocampus-dependent memory in aged (24-month-old), but not young (3month-old), F344xBN rats. More recently, we have demonstrated that this infection-evoked memory deficit is mirrored by a selective deficit in long-lasting synaptic plasticity in the hippocampus. Interestingly, these deficits occur in forms of long-term memory and synaptic plasticity known to be strongly dependent on brain-derived neurotrophic factor (BDNF). Here, we begin to test the hypothesis that the combination of aging and an infection might disrupt production or processing of BDNF protein in the hippocampus, decreasing the availability of BDNF for plasticity-related processes at synaptic sites. We find that mature BDNF is markedly reduced in Western blots of hippocampal synaptoneurosomes prepared from aged animals following infection. This reduction is blocked by intra-cisterna magna administration of the anti-inflammatory cytokine IL-1Ra (interleukin 1-specific receptor antagonist). Levels of the pan-neurotrophin receptor $\mathrm{p} 75^{\mathrm{NTR}}$ and the BDNF receptor TrkB (tropomyosin receptor kinase B) are not significantly altered in these synaptoneurosomes, but phosphorylation of TrkB and downstream activation of PLC $\gamma 1$ (phospholipase C $\gamma 1$ ) and ERK (extracellular response kinase) are attenuated-observations consistent with reduced availability of mature BDNF to activate TrkB signaling. These data suggest that inflammation-evoked reductions in BDNF at synapses might contribute to inflammation-evoked disruptions in long-term memory and synaptic plasticity in aging.

\section{Introduction}

Aging is associated with increased variability in cognitive functioning, in part because aging brains are more vulnerable to negative life events such as infections, surgery, and stress (Wofford et al., 1996; Bekker and Weeks, 2003; VonDras et al., 2005). We have recently developed a rodent model to study the mechanisms involved in this vulnerability (Barrientos et al., 2006). Twentyfour-month-old Fischer-Brown Norway rats are aged, but not senescent; they generally do not show significant physical or cognitive impairments. However, in response to signals triggered by activation of the peripheral innate immune system (by an intraperitoneal injection of Escherichia coli), they show an exaggerated inflammatory response in the brain. Following the immune challenge, hippocampal production of the proinflammatory cytokine

Received Nov. 4, 2010; revised Jan. 10, 2011; accepted Jan. 26, 2011.

This work was supported by an Innovative Seed Grant Award from the University of Colorado (to S.L.P.) and National Institute on Aging Grants 1R21AG031467 (to S.L.P.), and 1R01AG02827 (to R.M.B. and S.F.M.). We thank R. K. Bachtell, T. R. Chapman, G. H. McClelland, and M. J. LaVoie for helpful discussions.

Correspondence should be addressed to Dr. Susan L. Patterson, Department of Psychology and Neuroscience, University of Colorado at Boulder, Mail Stop 345-UCB, Boulder, C0, 80309. E-mail: susan.patterson@colorado.edu. DOI:10.1523/JNEUROSCI.5818-10.2011

Copyright $@ 2011$ the authors $\quad 0270-6474 / 11 / 314274-06 \$ 15.00 / 0$ interleukin- $1 \beta(\mathrm{IL}-1 \beta)$ is potentiated and prolonged in the aging rats relative to their younger (3-month-old) counterparts (Barrientos et al., 2009a). This exaggerated elevation in IL- $1 \beta$ does not compromise initial learning or formation of short-term memories, nor does it disrupt basal synaptic function or short-term synaptic plasticity - instead it is paralleled by specific deficits in hippocampus-dependent long-term memory tasks (e.g., contextual fear and place learning) and theta burst-evoked late-phase long-term potentiation (L-LTP) (Barrientos et al., 2009a; Chapman et al., 2010). Conversely, blocking IL-1 signaling in the brain with IL-1-specific receptor antagonist (IL-1Ra) ameliorates the deficits in memory (Frank et al., 2010) and L-LTP (Chapman et al., 2010).

It is not clear how aberrantly elevated levels of IL- $1 \beta$ in the hippocampus may produce limited, selective impairments in long-lasting forms of synaptic plasticity and memory. However, one intriguing possibility is suggested by the observation that infusion of IL- $1 \beta$ into the hippocampus decreases its capacity for transcription of brain-derived neurotrophic factor (BDNF) (Barrientos et al., 2004), and infusion of IL-1Ra protects it (Barrientos et al., 2003). BDNF plays a critical role in forms of long-lasting synaptic plasticity thought to be associated with consolidation of 
hippocampus-dependent memory (Tyler et al., 2002; Chao, 2003; Lu, 2003; Bramham and Messaoudi, 2005).

$\mathrm{BDNF}$ is synthesized as a precursor protein proBDNF that is post-translationally cleaved to produce mature BDNF (mBDNF). This processing of the BDNF protein appears to play a key role in determining its cellular functions (Barker, 2009; Greenberg et al., 2009). Pro-BDNF binds preferentially to the pan-neurotrophin receptor $\mathrm{p} 75^{\mathrm{NTR}}$, activates apoptosis-related signaling pathways, and may facilitate long-term depression in the hippocampus. In contrast, cleaved mBDNF binds to TrkB (tyrosine kinase B) tyrosine kinase receptors, promotes cell survival, and facilitates some forms of long-term potentiation.

In the experiments presented here, we have examined the combined effects of aging and an infection on levels of pro- and mature BDNF protein isoforms and their receptors in the hippocampus. The infection appears to produce limited, relatively subtle synaptic deficits rather than large-scale, nonspecific disruptions in hippocampal function (Chapman et al., 2010), and there is increasing evidence that some important pro-plasticity effects of BDNF are exerted locally at synapses (Kang and Schuman, 1996; Schratt et al., 2004). We have therefore prepared hippocampal synaptoneurosomes, enriching for perisynaptic proteins, and increasing the probability of detecting subtle changes in proteins at synaptic sites.

\section{Materials and Methods}

Animals. The rats used were 3- and 24-month old male Fischer344/ Brown Norway F1 crosses from the National Institute on Aging Aged Rodent Colony. Animals were pair housed, on a $12 \mathrm{~h}$ light/dark cycle, with ad libitum access to food and water. Upon arrival, rats were allowed to acclimate to the animal facility for 2 weeks before experiments were begun. All experiments conformed to protocols approved by the University of Colorado Animal Care and Use Committee.

E. coli infection model. Stock E. coli cultures (ATCC 15746; American Type Culture Collection) were thawed and cultured overnight in $40 \mathrm{ml}$ of brain-heart infusion (Difco) in an incubator $\left(37^{\circ} \mathrm{C}, 95 \%\right.$ air plus $5 \%$ $\mathrm{CO}_{2}$ ). The bacterial content in individual cultures was quantified by extrapolating from previously determined growth curves. Cultures were centrifuged for $15 \mathrm{~min}$ at $3000 \mathrm{rpm}$, the supernatants were discarded, and the bacteria were resuspended in sterile PBS, yielding a final dose of $2.5 \times$ $10^{9}$ colony-forming units in $250 \mu \mathrm{l}$. All animals received an intraperitoneal injection of $250 \mu \mathrm{l}$ of either $E$. coli or the vehicle (sterile PBS).

Blocking CNS consequences of the peripheral infection. IL-1Ra was injected into the cisterna magna, rather than into the cerebral ventricles or the hippocampus, because this procedure does not require surgery (which can itself produce memory impairments in aging animals). Rats were briefly anesthetized with halothane. The dorsal aspect of the skull was shaved and swabbed with $70 \% \mathrm{EtOH}$; then a 27 gauge needle attached via PE50 tubing to a $25 \mu \mathrm{l}$ Hamilton syringe was inserted into the cisterna magna. The IL-1Ra (112 $\mu \mathrm{g}$; Amgen) by intra-cisterna magna administration in a total volume of $3 \mu \mathrm{l}$; the animals received an intraperitoneal injection of either $E$. coli or vehicle immediately after.

Synaptoneurosome preparation. All tissue was collected $5 \mathrm{~d}$ after the injections. This time point was selected because of the following: (1) all of the animals have completely recovered from the acute infection (e.g., fever has subsided) (Barrientos et al., 2009b); (2) the aging, but not the young E. coli-injected rats show significant impairments in long-term memory (Barrientos et al., 2006) and L-LTP (Chapman et al., 2010); and (3) levels of IL-1 protein in the hippocampus are still significantly elevated in the aging rats, but not in the young rats (Barrientos et al., 2009a).

Rats underwent rapid decapitation, and hippocampi were extracted. Tissue was minced in $500 \mu$ l of homogenization buffer (HB) with protease and phosphatase inhibitors [ $1 \mathrm{~m}$ Tris, $1 \mathrm{~m}$ sucrose, $0.5 \mathrm{M}$ EDTA, $0.25 \mathrm{~m}$ EGTA, 0.5 M NaF, 1 m benzamidine, and 100 mM AEBSF (4-(2-aminoethyl)benzenesulfonyl fluoride)] and homogenized using a glass tissue grinder with a Teflon pestle. Nuclear material and unbroken cells were removed by centrifugation at $960 \times g$ for $15 \mathrm{~min}$. The remaining supernatant was centrifuged at 10,000 $\times g$ for 15 min, yielding an S2 cytosolic fraction and a P2 crude synaptoneurosomal fraction containing both presynaptic and postsynaptic material. The P2 synaptoneurosomal pellet was washed gently in $100 \mu \mathrm{l}$ of HB. The P2 pellet was then homogenized using a $0.5 \mathrm{ml}$ plastic pestle in $100 \mu \mathrm{l}$ of $\mathrm{HB}$ with $10 \mu \mathrm{l}$ of $10 \times$ sodium chloride-TRIS-EDTA $(1 \times$ final concentration) and sonicated. The $\mathrm{P} 2$ fraction obtained using this protocol is enriched for perisynaptic components including presynaptic and postsynaptic proteins, terminal mitochondria and cytoplasm and synaptic vesicles (Booth and Clark, 1978; Whittaker, 1993). Synaptic enrichment of the $\mathrm{P} 2$ fraction was confirmed using synaptophysin and postsynaptic density 95 (PSD95), common synaptic markers. Protein content was quantified using the BCA protein assay (Bio-Rad).

Western blots. Samples were prepared under reducing conditions in $4 \times$ Laemmli buffer and heated at $70^{\circ} \mathrm{C}$ for $5 \mathrm{~min}$. For Western blotting, $40 \mu \mathrm{g}$ of protein sample was loaded onto $4-12 \%$ NuPage (Invitrogen) Bis-Tris SDS-polyacrylamide gels and transferred onto polyvinylidene fluoride membranes (Millipore). Membranes were blocked in 5\% milk/ PBST (PBS with Triton) for $30 \mathrm{~min}$ at room temperature; all primary antibody incubations were at $4^{\circ} \mathrm{C}$ overnight followed by $3 \times 10 \mathrm{~min}$ washes with PBST; secondary antibody incubations were at room temperature for $1 \mathrm{~h}$ and washed $3 \times 10 \mathrm{~min}$. The following primary antibodies (and dilutions) were used: mature BDNF (1:1000; sc-546; Santa Cruz Biotechnology), proBDNF (1:500; ab72440; Abcam), phospho-TrkB (1: 700; pTrkBY816, antisera gift from Moses Chao, New York University School of Medicine, New York, NY) and total TrkB (1:1000; sc-8316; Santa Cruz Biotechnology), p75 (1:500; gift from Mark Bothwell, University of Washington, Seattle, WA), phospho-PLC $\gamma 1$ (phospholipase C $\gamma 1)(1: 1000 ; 07-2134)$ and total (phosphorylated and unphosphorylated) PLC $\gamma 1$ (1:500; 05-366; Millipore), phospho-ERK (extracellular response kinase) $(1: 1000 ; 9101)$ and total ERK $(1: 1000 ; 9102$; Cell Signaling Technology), and phospho-AKT $(1: 1000 ; 4058)$ and total AKT (1: 1000; 9272; Cell Signaling Technology). Blots were probed with synaptic markers synaptophysin (1:1000; sc-12737; Santa Cruz Biotechnology) and PSD95 (1:1000; United Biomedical) to validate synaptoneurosome fractions, and $\beta$-tubulin (1:100,000; MAB1637; Millipore Bioscience Research Reagents) and $\beta$-actin (1:5000; sc-47778; Santa Cruz Biotechnology) as loading controls. Identity of the BDNF isoform bands in synaptoneurosomes was confirmed by comparison with extracts from HeLa cells transfected with a plasmid that overexpresses BDNF, producing both the pro- and mature form. Secondary antibodies were purchased from GE Healthcare and Bio-Rad, and were diluted in the range of 1:5000-1:10000; SuperSignal West Pico Chemiluminescent was purchased from Pierce. Following chemiluminescent application, blots were exposed to autoradiography film (Denville Scientific). Blots were stripped using Restore Western Blot Stripping Buffer (Pierce) for $15 \mathrm{~min}$, washed $3 \times 10 \mathrm{~min}$ in PBST, and subjected to standard Western blotting conditions.

Analysis. Protein bands were quantified using ImageJ software, and all bands were normalized to their actin controls. Because we had previously shown that the combination of age and infection uniquely disrupts BDNF-dependent plasticity and memory, we hypothesized that it might also uniquely reduce BDNF (and related proteins). We therefore used an unpaired $t$ test to determine whether the level of the protein of interest in the aged $+E$. coli group differed from the level of the protein in the other groups. The $p$ value listed for each protein (or phosphorylation state ratio) is for an unpaired $t$ test comparing the mean of the aging $+E$. coli group to the mean of the summed values of the other test groups.

\section{Results}

Levels of the mature BDNF protein isoform are significantly reduced in hippocampal synaptoneurosomes prepared from aged animals with a recent history of infection

We hypothesized that aging and a recent history of infection might interact to disrupt biosynthesis or processing of BDNF protein in the hippocampus, decreasing the availability of BDNF for plasticity-related processes at synaptic sites. To begin investigating this possibility, we prepared synaptoneurosomes from 
hippocampi collected from young and aging rats $5 \mathrm{~d}$ after a vehicle or $E$. coli injection. This procedure produces a significant enrichment in synaptic proteins, making it possible to identify subtle experience-dependent changes in the protein composition of synapses (Whittaker, 1993).

Western blot analysis detected a specific proBDNF signal (ab72440 antibody; Abcam) in the $30-35 \mathrm{kDa}$ range (Fig. $1 B$ ). The combination of age and infection produced a trend toward a slight (10$15 \%)$ reduction in the proBDNF signal, but it did not reach significance $\left(t_{(18)}=\right.$ $1.234, p=0.232$ ). In contrast, further analysis with an antibody for the mature domain of BDNF (sc-546 antibody; Santa Cruz Biotechnology) (Lee et al., 2001) revealed that age and infection together reduced levels of mBDNF by more than half $\left(t_{(22)}=2.615, p=0.0158\right)$ (Fig. $1 C$ ).

\section{Levels of receptors for BDNF are not significantly altered by aging or a history of infection}

In contrast to its effects on BDNF protein, the combination of age and infection produced no detectable changes in levels of BDNF receptors in hippocampal synaptoneurosomes prepared $5 \mathrm{~d}$ after the initial E. coli injection. Expression of the p $75^{\text {NTR }}$ receptor was unchanged (antibody gift from Mark Bothwell, University of Washington, Seattle, WA) (Fig. 2B). Similarly, total levels of TrkB did not vary significantly across conditions, nor was there a shift in the relative proportions of full-length vs truncated (lacking the tyrosine kinase) TrkB receptor isoforms (sc-8316; Santa Cruz Biotechnology) (Fig. 2A).

\section{Age and infection interact to reduce activation of TrkB and downstream signaling systems}

Activation of TrkB by mBDNF triggers a series of phosphorylation events, beginning with the receptor, which can activate proteins in the three major growth factor-regulated signaling pathways: the PLC $\gamma 1$ pathway, the Ras/ERK pathway, and the PI3K (phosphatidyl inositol 3-kinase)/Akt pathway (Greene and Kaplan, 1995; Huang and Reichardt, 2003). We found that the ratio of phospho-TrkB (antisera gift from Moses Chao) to total TrkB (sc-8316; Santa Cruz Biotechnology) was significantly reduced by the combination of age and infection $\left(t_{(14)}=4.680, p=0.0004\right)($ Fig. $3 A)$. We next asked whether this was associated with reduced activation of PLC $\gamma 1$, ERK, and or Akt. We found that the ratio of phosphoPLC- $\gamma 1$ (07-2134; Millipore) to total PLC- $\gamma 1$ (05-366; Millipore) was significantly reduced in synaptoneurosomes from aged

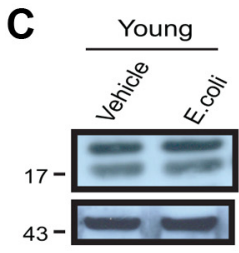

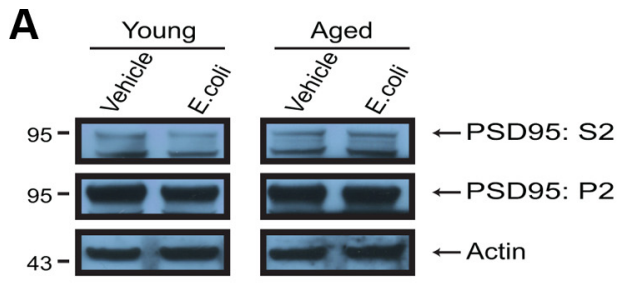
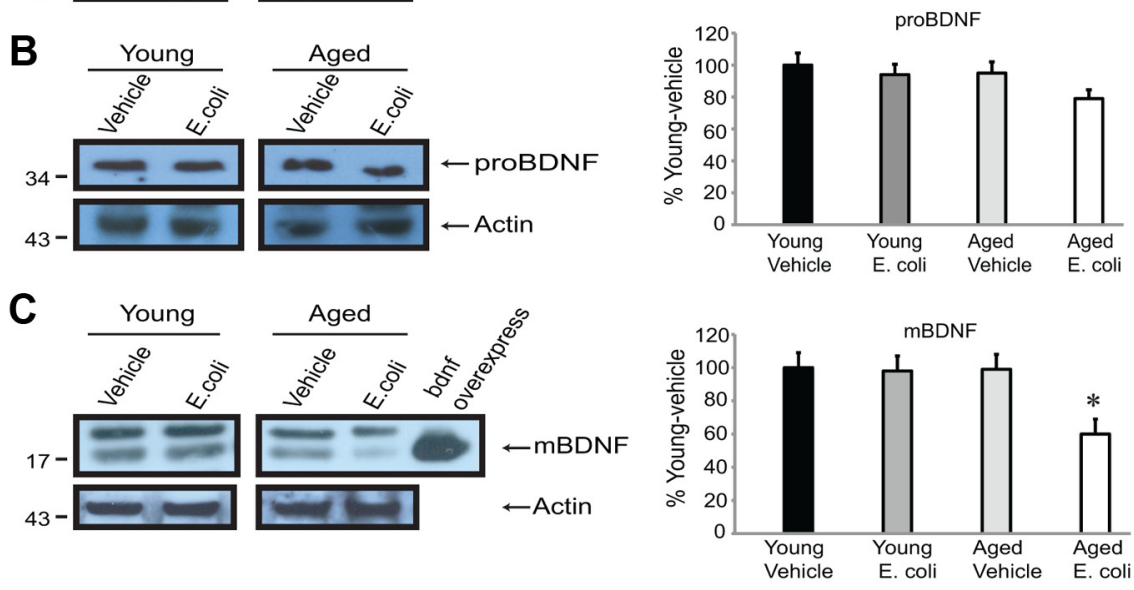

Figure 1. Infection differentially affects BDNF protein isoforms in aged versus young animals. Western blot analysis showing enrichment of synaptic material in synaptoneurosomes, and levels of proBDNF and mature BDNF in hippocampal synaptoneurosomes prepared from young and aged rats, with and without a recent history of infection. $A$, Representative examples of blots probed with a PSD95 antibody to confirm enrichment of synaptic material in the synaptoneurosomal (P2) versus the cytosolic (S2) fractions. $\boldsymbol{B}$, Levels of proBDNF were not significantly reduced by the combination of aging and infection. $\boldsymbol{C}$, Infection markedly reduced mature BDNF in synaptic fractions from aged animals. Band intensities were quantified (ImageJ), normalized to actin controls, and expressed as percentages of mean protein levels from young vehicle-injected rats. Error bars indicate SEM. All graphs (here and below) represent a minimum of three independent experiments with one to two animals per group in each experiment. Asterisks indicate statistical significance from all other groups; individual $p$ values are reported in the text.
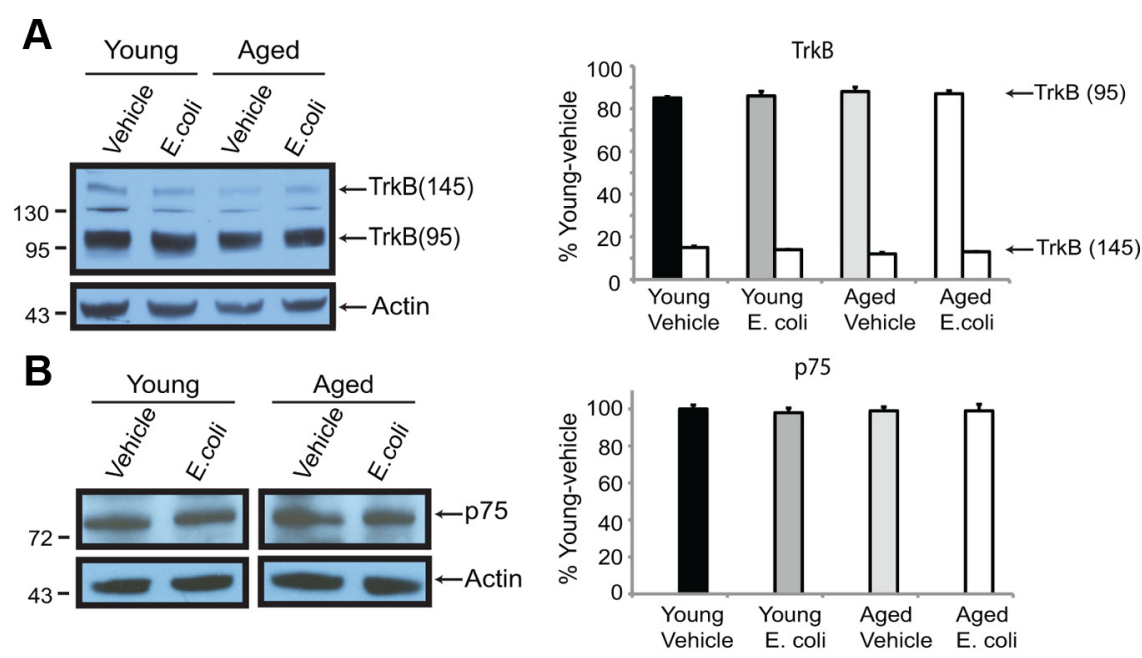

Figure 2. Age and infection do not alter levels of BDNF receptors under the conditions of the study. Western blot analysis was performed on hippocampal synaptoneurosomes prepared from young and aged rats, with and without a recent history of infection. $\boldsymbol{A}, \boldsymbol{B}$, Levels of the pan neurotrophin receptor p $75^{\mathrm{NTR}}(\boldsymbol{A})$ and principal TrkB receptor isoforms $(\boldsymbol{B})$ were unchanged. Quantification was as described in the Fig. 1 legend.

rats following infection $\left(t_{(10)}=4.468, p=0.0012\right)$ (Fig. 3B). This was also true of the ratio of phospho-ERK (9101; Cell Signaling Technology) to total ERK (9102; Cell Signaling Technology) $\left(t_{(10)}=5.581, p=0.0002\right.$ ) (Fig. 3C). In contrast, we found that the ratio of phospho-Akt to total Akt was not significantly reduced under the conditions examined $\left(t_{(10)}<1, p=0.6568\right)$ (Fig. 3D). 

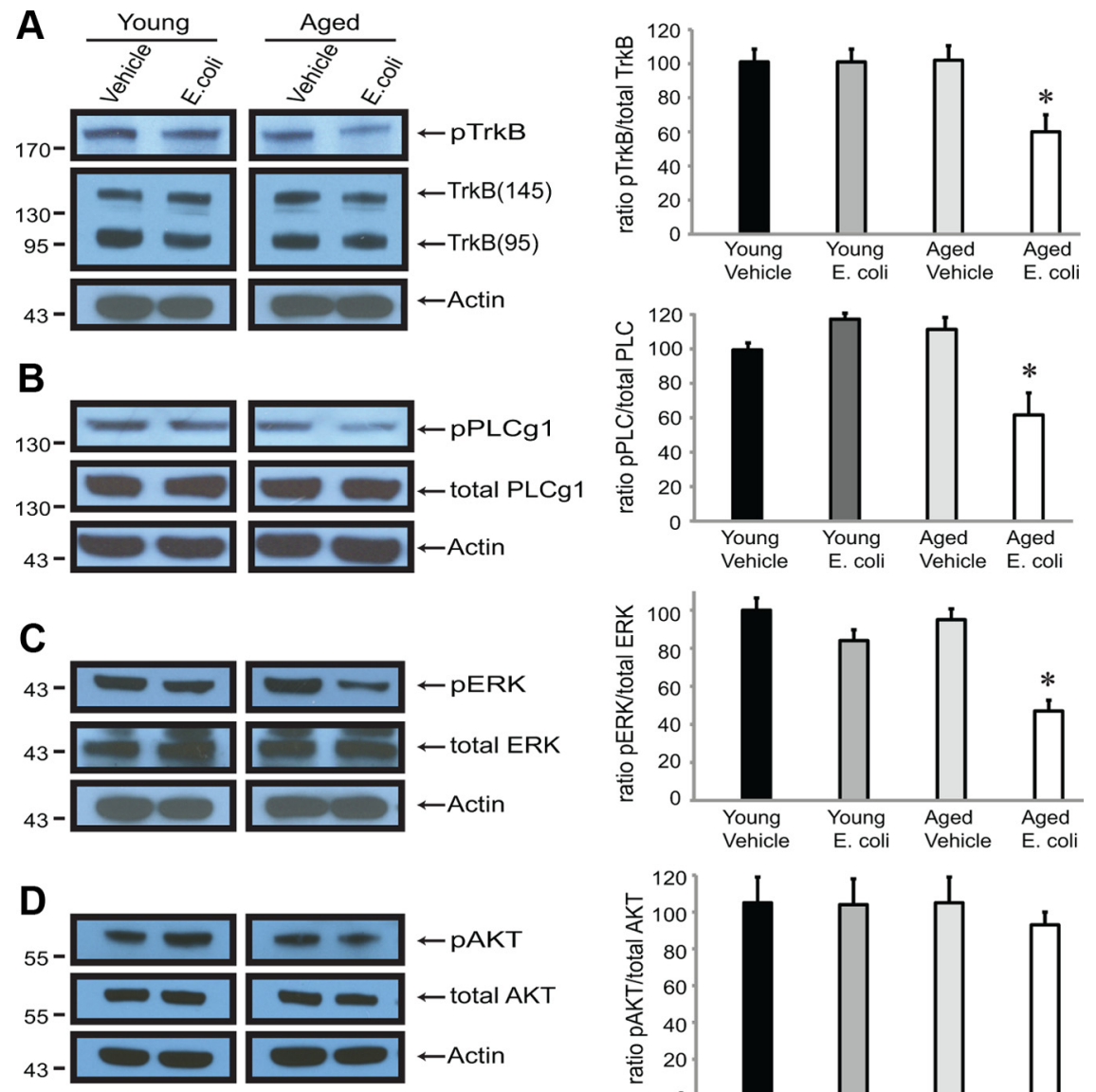

Central administration of the anti-inflammatory cytokine IL-1Ra ameliorates the infection-induced reductions in $\mathrm{mBDNF}$ and activated TrkB in synaptoneurosomes from aged rats

IL- $1 \beta$ is a major mediator of inflammatory responses in the brain as well as in the periphery. We have previously shown that blocking the actions of IL- $1 \beta$ in the brain, by injecting IL-1Ra into the cisterna magna at the time of the intraperitoneal $E$. coli injection, blocks infection-evoked deficits in long-term synaptic plasticity and memory in aged rats (Chapman et al., 2010; Frank et al., 2010). Here we report that administration of IL-1Ra also blocks $E$. coli-evoked reductions in $\operatorname{mBDNF}\left(t_{(10)}=0.3511, p=0.7328\right)$ (Fig. $4 A)$ and phospho-TrkB $\left(t_{(10)}=\right.$ 1.339, $p=0.2102$ ) (Fig. $4 B$ ).

\section{Discussion}

We have previously demonstrated that a peripheral immune challenge produces profound disruptions in forms of hippocampus-dependent long-term memory and synaptic plasticity known to be BDNF dependent in aged, but not young, F344xBN rats (Barrientos et al., 2006; Chapman et al., 2010). Here, we have extended these observations, examining for the first time the combined effects of aging and infection on levels of BDNF protein isoforms and their receptors at synaptic sites in the hippocampus. Our key findings are that an immune challenge in aging rats (1) triggered a minimal reduction in proBDNF and a much larger reduction in mature BDNF detectable in hippocampal synaptoneurosomes prepared $5 \mathrm{~d}$ after the injections, after all the rats had recovered from the acute infection; (2) had no significant effects on levels of BDNF receptors; but (3) significantly reduced phosphorylation of TrkB, and downstream activation of PLC $\gamma 1$ and ERK, consistent with decreased availability of mBDNF for activation of TrkB; and (4) no longer reduced $\mathrm{mBDNF}$ and phospho-TrkB if IL-1 receptors in the brain were blocked with a selective antagonist.

These new data are consistent with the hypothesis that the interaction of aging and an infection might decrease availability of BDNF at hippocampal synapses, and thus might contribute to selective deficits in forms of long-lasting plasticity and memory that require BDNF for their full expression. We found that the interaction of aging and infection reduced levels of $\mathrm{mBDNF}$ at synaptic sites by $>50 \%$. Mouse models of BDNF haploinsuffi-
Figure 4. $\quad \boldsymbol{A}, \boldsymbol{B}$, Blocking IL-1 $\beta$ signaling in the CNS blocks the $E$. coli-evoked reduction in synaptic levels of $m B D N F$ protein $(\boldsymbol{A})$ and phospo-TrkB in aged rats $(\boldsymbol{B})$. E. coli-injected rats received a concurrent injection of the anti-inflammatory cytokine IL-1Ra or vehicle into the cisterna magna. Hippocampi were collected, and synaptoneurosomes prepared $5 \mathrm{~d}$ after the injections. Quantification was as described in the Fig. 1 legend. 
ciency have provided evidence that a critical threshold level of BDNF is necessary for full function in memory-related plasticity. Heterozygous BDNF $(+/-)$ mice with approximately half the normal levels of BDNF in their brains have significant impairments in long-lasting synaptic plasticity (Korte et al., 1995; Patterson et al., 1996; Pang et al., 2004) and in hippocampus-dependent learning and memory (Linnarsson et al., 1997).

The consequences of reduction of BDNF protein isoforms are not yet so well studied, but mBDNF appears to play an important role in some forms of long-lasting synaptic plasticity in the hippocampus. Mature BDNF can be generated by cleavage of proBDNF by plasmin, an extracellular protease activated by tissue plasminogen activator (tPA)-dependent cleavage of plasminogen (Pang et al., 2004). Theta burst stimulation is reported to induce secretion of tPA, and to increase extracellular conversion of proBDNF to mBDNF in cultures of hippocampal neurons (Nagappan et al., 2009). Application of mBDNF, but not a cleavage-resistant proBDNF, can rescue deficits in theta burst L-LTP hippocampal slices from mice lacking tPA or plasminogen (Pang et al., 2004). Mature BDNF can also rescue the impairment of theta-burst L-LTP caused by inhibition of protein synthesis in wild-type mice (Pang et al., 2004), suggesting that the mBDNF isoform may be one of the proteins whose production is required for long-lasting enhancement of synaptic efficacy. There is now some corresponding evidence that production of adequate amounts of mBDNF may be important for hippocampusdependent memory. A recent study indicates that increased cleavage of precursor proBDNF in the hippocampus is positively correlated with acquisition of contextual fear memory, while decreased cleavage is associated with extinction (Barnes and Thomas, 2008).

The effects of aging on BDNF mRNA and protein have been extensively studied and generally found to be modest (Pang and $\mathrm{Lu}, 2004)$. However, a few studies have now examined the impact of aging on BDNF protein isoforms. Aged Wistar rats with memory impairments are reported to have lower levels of total BDNF, but higher ratios of proBDNF to $\mathrm{mBDNF}$ than controls from a related strain known to have better preservation of cognitive function (Silhol et al., 2008). Perhaps not surprisingly, training in a spatial learning task increased levels of proBDNF in both young and aged Wistar rats, but only the young rats showed a corresponding increase in mBDNF (Silhol et al., 2007).

Several studies have examined the effects of immune challenge or proinflammatory cytokines on BDNF in the brain. Lipopolysaccharide (LPS) is a component of the cell wall of Gram-negative bacteria. A potent endotoxin, it leads to the release of proinflammatory cytokines such as IL- $1 \beta$ and tumor necrosis factor $\alpha$ (Drum and Oppemtom, 1989). BDNF mRNA in the principle neurons of the hippocampus was strongly downregulated $4 \mathrm{~h}$ after an intraperitoneal injection of LPS or IL-1 $\beta$ (Lapchak et al., 1993). Depolarization-induced release of BDNF from slices of dentate gyrus was not altered by administration of LPS several days earlier (Shaw et al., 2001). However, when levels of BDNF protein were examined $7 \mathrm{~h}$ after intraperitoneal injection of LPS, LPS was found to produce a dose-dependent reduction in BDNF in the cortex and hippocampus (Guan and Fang, 2006).

Much less is known about the effects of immune challenge on BDNF protein isoforms, but intraperitoneal injection of a single very high dose of LPS $(3 \mathrm{mg} / \mathrm{kg})$ is reported to produce a small $(\sim 15 \%)$ reduction in both proBDNF and mature BDNF in crude synaptoneurosomes prepared from the brains of young mice $3 \mathrm{~d}$ after the injection (Schnydrig et al., 2007). We have previously shown that a peripheral $E$. coli infection produces an exaggerated inflammatory response in the brains of aging animals, paralleled by specific deficits in forms of long-term memory and synaptic plasticity known to be strongly dependent on BDNF (Barrientos et al., 2009a,b; Chapman et al., 2010). We now report that this inflammation also gives rise to a large reduction in $\mathrm{mBDNF}$ and TrkB signaling at synapses in the hippocampus.

It is not yet clear how exaggerated CNS inflammation may impact levels of BDNF protein isoforms. The precise circumstances and sites of BDNF production, processing, and release, and the stability of the resulting isoforms is currently a very active, complex, and controversy-filled area of investigation (Barker, 2009)—and rather beyond the scope of this initial report. However, the work presented here provides new insights into naturalistic events that can affect BDNF production and processing in vivo and should provide a basis for further investigation of the interactions of aging, inflammation, and BDNF biology.

\section{References}

Barker PA (2009) Whither proBDNF? Nat Neurosci 12:105-106.

Barnes P, Thomas KL (2008) Proteolysis of proBDNF is a key regulator in the formation of memory. PLoS One 3:e3248.

Barrientos RM, Sprunger DB, Campeau S, Higgins EA, Watkins LR, Rudy JW, Maier SF (2003) Brain-derived neurotrophic factor mRNA downregulation produced by social isolation is blocked by intrahippocampal interleukin-1 receptor antagonist. Neuroscience 121:847-853.

Barrientos RM, Sprunger DB, Campeau S, Watkins LR, Rudy JW, Maier SF (2004) BDNF mRNA expression in rat hippocampus following contextual learning is blocked by intrahippocampal IL-1beta administration. J Neuroimmunol 155:119-126.

Barrientos RM, Higgins EA, Biedenkapp JC, Sprunger DB, Wright-Hardesty KJ, Watkins LR, Rudy JW, Maier SF (2006) Peripheral infection and aging interact to impair hippocampal memory consolidation. Neurobiol Aging 27:723-732.

Barrientos RM, Frank MG, Hein AM, Higgins EA, Watkins LR, Rudy JW, Maier SF (2009a) Time course of hippocampal IL-1 beta and memory consolidation impairments in aging rats following peripheral infection. Brain Behav Immun 23:46-54.

Barrientos RM, Watkins LR, Rudy JW, Maier SF (2009b) Characterization of the sickness response in young and aging rats following E. coli infection. Brain Behav Immun 23:450-454.

Bekker AY, Weeks EJ (2003) Cognitive function after anaesthesia in the elderly. Best Pract Res Clin Anaesthesiol 17:259-272.

Booth RF, Clark JB (1978) A rapid method for the preparation of relatively pure metabolically competent synaptosomes from rat brain. Biochem J 176:365-370.

Bramham CR, Messaoudi E (2005) BDNF function in adult synaptic plasticity: the synaptic consolidation hypothesis. Prog Neurobiol 76:99-125.

Chao MV (2003) Neurotrophins and their receptors: a convergence point for many signalling pathways. Nat Rev Neurosci 4:299-309.

Chapman TR, Barrientos RM, Ahrendsen JT, Maier SF, Patterson SL (2010) Synaptic correlates of increased cognitive vulnerability with aging: peripheral immune challenge and aging interact to disrupt theta-burst latephase long-term potentiation in hippocampal area CA1. J Neurosci 30:7598-7603.

Drum SK, Oppemtom JJ (1989) Macrophage derived mediators: IL1, TNF, IL6, IGN and related signals. In: Fundamental immunology (Paul WE, ed), pp 639-661. New York: Raven.

Frank MG, Barrientos RM, Hein AM, Biedenkapp JC, Watkins LR, Maier SF (2010) IL-1RA blocks E. coli-induced suppression of Arc and long-term memory in aged F344xBN F1 rats. Brain Behav Immun 24:254-262.

Greenberg ME, Xu B, Lu B, Hempstead BL (2009) New insights in the biology of BDNF synthesis and release: implications in CNS function. J Neurosci 29:12764-12767.

Greene LA, Kaplan DR (1995) Early events in neurotrophin signalling via Trk and p75 receptors. Curr Opin Neurobiol 5:579-587.

Guan Z, Fang J (2006) Peripheral immune activation by lipopolysaccharide decreases neurotrophins in the cortex and hippocampus in rats. Brain Behav Immun 20:64-71. 
Huang EJ, Reichardt LF (2003) Trk receptors: roles in neuronal signal transduction. Annu Rev Biochem 72:609-642.

Kang H, Schuman EM (1996) A requirement for local protein synthesis in neurotrophin-induced hippocampal synaptic plasticity. Science 273:1402-1406.

Korte M, Carroll P, Wolf E, Brem G, Thoenen H, Bonhoeffer T (1995) Hippocampal long-term potentiation is impaired in mice lacking brainderived neurotrophic factor. Proc Natl Acad Sci U S A 92:8856-8860.

Lapchak PA, Araujo DM, Hefti F (1993) Systemic interleukin-1 beta decreases brain-derived neurotrophic factor messenger RNA expression in the rat hippocampal formation. Neuroscience 53:297-301.

Lee R, Kermani P, Teng KK, Hempstead BL (2001) Regulation of cell survival by secreted proneurotrophins. Science 294:1945-1948.

Linnarsson S, Björklund A, Ernfors P (1997) Learning deficit in BDNF mutant mice. Eur J Neurosci 9:2581-2587.

Lu B (2003) BDNF and activity-dependent synaptic modulation. Learn Mem 10:86-98.

Nagappan G, Zaitsev E, Senatorov VV Jr, Yang J, Hempstead BL, Lu B (2009) Control of extracellular cleavage of ProBDNF by high frequency neuronal activity. Proc Natl Acad Sci U S A 106:1267-1272.

Pang PT, Lu B (2004) Regulation of late-phase LTP and long-term memory in normal and aging hippocampus: role of secreted proteins tPA and BDNF. Ageing Res Rev 3:407-430.

Pang PT, Teng HK, Zaitsev E, Woo NT, Sakata K, Zhen S, Teng KK, Yung WH, Hempstead BL, Lu B (2004) Cleavage of proBDNF by tPA/plasmin is essential for long-term hippocampal plasticity. Science 306:487-491.

Patterson SL, Abel T, Deuel TA, Martin KC, Rose JC, Kandel ER (1996) Recombinant BDNF rescues deficits in basal synaptic transmission and hippocampal LTP in BDNF knockout mice. Neuron 16:1137-1145.

Schnydrig S, Korner L, Landweer S, Ernst B, Walker G, Otten U, Kunz D
(2007) Peripheral lipopolysaccharide administration transiently affects expression of brain-derived neurotrophic factor, corticotropin and proopiomelanocortin in mouse brain. Neurosci Lett 429:69-73.

Schratt GM, Nigh EA, Chen WG, Hu L, Greenberg ME (2004) BDNF regulates the translation of a select group of mRNAs by a mammalian target of rapamycin-phosphatidylinositol 3-kinase-dependent pathway during neuronal development. J Neurosci 24:7366-7377.

Shaw KN, Commins S, O’Mara SM (2001) Lipopolysaccharide causes deficits in spatial learning in the watermaze but not in BDNF expression in the rat dentate gyrus. Behav Brain Res 124:47-54.

Silhol M, Arancibia S, Maurice T, Tapia-Arancibia L (2007) Spatial memory training modifies the expression of brain-derived neurotrophic factor tyrosine kinase receptors in young and aged rats. Neuroscience 146:962-973.

Silhol M, Arancibia S, Perrin D, Maurice T, Alliot J, Tapia-Arancibia L (2008) Effect of aging on brain-derived neurotrophic factor, proBDNF, and their receptors in the hippocampus of Lou/C rats. Rejuvenation Res 11:1031-1040.

Tyler WJ, Alonso M, Bramham CR, Pozzo-Miller LD (2002) From acquisition to consolidation: on the role of brain-derived neurotrophic factor signaling in hippocampal-dependent learning. Learn Mem 9:224-237.

VonDras DD, Powless MR, Olson AK, Wheeler D, Snudden AL (2005) Differential effects of everyday stress on the episodic memory test performances of young, mid-life, and older adults. Aging Ment Health 9:60-70.

Whittaker VP (1993) Thirty years of synaptosome research. J Neurocytol 22:735-742.

Wofford JL, Loehr LR, Schwartz E (1996) Acute cognitive impairment in elderly ED patients: etiologies and outcomes. Am J Emerg Med 14:649653. 